\title{
Recognition of tissue cyst-specific antigens in reactivating toxoplasmosis
}

\author{
T. D. MchUGH, T. BATHGATE*, J. MANGAN*, J. D. JOHNSON*, R. E. HOLLIMAN* and \\ P. D. BUTCHER*
}

Department of Medical Microbiology, Royal Free Hospital School of Medicine, Pond Street, London NW3 206 and *Department of Medical Microbiology and PHLS Toxoplasma Reference Unit, St George's Hospital Medical School, Cranmer Terrace, London SW17 ORE

Current serological tests do not discriminate between asymptomatic latent Toxoplasma gondii infection and reactivating toxoplasmosis, but timely therapeutic intervention before the development of symptoms would lead to major reductions in morbidity and permanent disability. This study developed a new enzyme-linked immunosorbent assay (ELISA) for antibody to $T$. gondii tissue cyst antigens and screened tissue cyst antigens by Western blot analysis to test the hypothesis that antibody recognition of $T$. gondii tissue cyst-derived antigen is a good indicator of reactivation disease. A total of 187 sera was tested by Sabin-Feldman dye test and tissue cyst ELISA. AIDS patients and patients with ocular disease were considered separately, as the exposure to parasite antigens may be different in these two groups. The dye test did not discriminate between immunocompetent and immunocompromised $T$. gondii seropositive patients or between active and quiescent toxoplasmosis. Tissue cyst ELISA demonstrated a raised specific antibody response in immunocompetent $T$. gondii seropositive patients and in quiescent HIV positive sera. These data support the view that the tissue cyst population is in a state of dynamic equilibrium. It is proposed that, in the immunocompetent host, tissue cyst development and rupture are under some degree of immune control, but that in the immunocompromised host this equilibrium is disturbed and reactivation disease results. Data from patients with reactivating ocular toxoplasmosis demonstrate that tissue cystspecific antibody levels are not different in active and quiescent disease and indeed they are not significantly different from immunocompetent $\boldsymbol{T}$. gondii seronegative sera. In the Western blot analysis of 57 HIV positive patient sera, eight antigens $(65,57,49,47,36$, 28,26 and $18 \mathrm{kDa}$ ) were consistently recognised by one third or more of the sera tested, but no single antigen was diagnostic of quiescent or active toxoplasmosis. It is concluded that tissue cyst-derived antigens are not a reliable serological marker of reactivating toxoplasmosis.

\section{Introduction}

Reactivation of the tissue cyst of Toxoplasma gondii is the major pathogenic event in the development of toxoplasmic encephalitis (TE) in AIDS patients and of ocular disease in individuals with congenitally acquired toxoplasmosis (CT). Definitive diagnosis of TE is rarely achieved and in AIDS patients follows the onset of symptoms associated with central nervous system (CNS) involvement. Definitive diagnosis in this patient group requires brain biopsy and either histological

Received 30 July 1996; revised version accepted 18 Nov. 1996.

Corresponding author: Dr T. D. McHugh. demonstration or culture of parasites [1]. A confirmed diagnosis of ocular toxoplasmosis requires extended ophthalmic supervision with prompt treatment in periods of acute exacerbation [2]. The anti-protozoal agents used in the treatment of these patient groups have toxic effects, precluding prolonged prophylactic use. Timely therapeutic intervention before the development of symptoms would be considered highly desirable, leading to major reductions in morbidity and permanent disability.

Serodiagnosis for monitoring of primary toxoplasmosis is well established; assays use the tachyzoite as the principal antigen [3]. However, these tests do not discriminate an asymptomatic latent infection from reactivating disease, even though both bradyzoite and 
tachyzoite stage specific [4] and shared [5] antigens are detectable during the development of an immune response in reactivating toxoplasmosis. In most AIDS patients serological evidence of previous $T$. gondii infection is seen before the onset of encephalitis. However, following onset of symptoms only a minority of patients with AIDS and TE demonstrate a significant change in anti-tachyzoite IgG or IgM antibody titre, or both [1]. Thus, a serological test capable of discriminating between active and quiescent toxoplasmosis in this population would provide a significant improvement in diagnosis and treatment. Suzuki and Remington [3] proposed that membranebound formalin-fixed tachyzoite antigens improved the serodiagnosis of TE in patients with AIDS. Their study discriminated between patients with TE demonstrated at biopsy and ambulatory patients asymptomatic for CNS diseases, but was not tested as a predictive assay for the onset of TE.

The hypothesis that antibody recognition of $T$. gondii tissue cyst-derived antigen (as distinct from tachyzoite-derived antigen) is a good indicator of reactivation disease was tested. The assumption is made that the reactivating tissue cyst presents both cyst wall/ matrix and bradyzoite-derived antigens to immune surveillance as well as tissue cyst-derived tachyzoite antigens [6]. This retrospective study of sera from patients with acute and quiescent toxoplasmosis considered AIDS patients and patients with ocular disease separately, as the exposure to parasite antigens may be different in these two groups. The hypothesis has been tested by the development of a new enzyme-linked immunosorbent assay (ELISA) for antibody to cyst antigens and screening of cyst antigens by Western blot analysis.

\section{Materials and methods}

\section{Patients}

Sera were selected from the PHLS Toxoplasma Reference Unit serum bank. Three groups were studied. (1) Immunocompetent patients with no ocular disease presenting for investigation of lymphadenopathy, monospot negative glandular fever or other non-specific disease; $T$. gondii sero-negative and $T$. gondii seropositive. (2) Patients with active or quiescent ocular toxoplasmosis and serologically confirmed exposure to the parasite. Active ocular disease was defined as those patients with evidence of inflammation on retinal examination. Quiescent ocular toxoplasmosis was defined as those patients with clinical evidence of retinal lesions characteristic of $T$. gondii infection in the absence of active inflammation (i.e., evidence of previous but not current reactivation). (3) HIV positive patients, with active cerebral toxoplasmosis or with quiescent toxoplasmosis. Active cerebral toxoplasmosis was defined as: compatible clinical presentation, SabinFeldman dye test positive, compatible computerised tomographic scan and subsequent response to specific therapy.

\section{Antigen preparations}

For preparation of T. gondii tissue cysts, Sau strain [7] was maintained in C57/BL10 mice as described by Roberts and Alexander [8]. After 4 weeks the mice were killed and brains were removed and homogenised in a Dounce homogeniser with $2 \mathrm{ml}$ of sterile phosphate-buffered saline (PBS). Tissue cysts were then purified on pre-formed Percoll gradients, $45 \% \mathrm{v} / \mathrm{v}$ in PBS (1250 g, $30 \mathrm{~min}$, room temperature) [9], washed three times and resuspended in PBS. Tissue cysts were counted and the preparation was stored as a pellet at $-70^{\circ} \mathrm{C}$. In the order of $20000-50000$ tissue cysts/ mouse brain were recovered. Complete rupture of tissue cysts was ensured before use; the pellet was resuspended in $500 \mu \mathrm{l}$ of distilled water and submitted to five cycles of freezing in a methanol-dry ice bath and thawing at $37^{\circ} \mathrm{C}$. The suspension was then sonicated at $50 \mathrm{cycles} / \mathrm{s}$ for $30 \mathrm{~s}$ on ice; tissue cysts were checked microscopically for rupture. Sonication was repeated until complete rupture was observed.

$\mathrm{RH}$ strain tachyzoites were passaged in MF1 mice as described previously [10]. Parasites were washed three times, resuspended in PBS, counted and stored as a pellet at $-70^{\circ} \mathrm{C}$.

\section{Quantitative serology}

All serum samples were assessed for the presence of $T$. gondii-specific antibody by the Sabin-Feldman dye test [11]. Seropositivity was defined as a dye test result of $\geqslant 2$ IU.

IgM was measured by a double sandwich ELISA (DSELISA) and an immunosorbent agglutination assay (ISAGA) [12].

An ELISA for the presence of $T$. gondii tissue cystspecific antibody was developed. Falcon flat-bottomed microtitration plates were coated at $4^{\circ} \mathrm{C}$ overnight in a humidity chamber with tissue cyst suspension diluted to 20 tissue cysts/well in $\mathrm{Na}_{2} \mathrm{CO}_{3} / \mathrm{NaHCO}_{3}$ coating buffer $(0.05 \mathrm{M}, \mathrm{pH} 9.6)$. Plates were washed once with PBS, then three times with PBST (PBS, Tween 20 $0.5 \%$ ) for $5 \mathrm{~min}$ each. Patient sera were diluted 1 in 1000 in PBST containing goat serum $1 \%$ and $100 \mu \mathrm{l} /$ well were incubated for $1 \mathrm{~h}$, at $37^{\circ} \mathrm{C}$ in a humidity chamber. Plates were washed three times with PBST for $5 \mathrm{~min}$ each and then incubated with $100 \mu \mathrm{l}$ of goat anti-human horseradish peroxidase conjugate (Sigma) diluted 1 in 10000 in goat serum in PBST $1 \%$ for $1 \mathrm{~h}$ at $37^{\circ} \mathrm{C}$ in a humidity chamber. Plates were washed four times with PBST for $5 \mathrm{~min}$ each before developing with tetramethylbenzidine (TMB) in acetate buffer $(0.1 \mathrm{M}$ sodium acetate, $0.1 \mathrm{M}$ citric acid, $\mathrm{pH} 6.0$ ) and hydrogen peroxide at a final 
concentration of $0.5 \% \mathrm{v} / \mathrm{v}$. Absorbance was measured at $450 \mathrm{~nm}$ on a Titretek Multiskan MCC/340 ELISA plate reader. Results were expressed as enzyme immunoassay units (EIU) calculated as described by Balfour [13].

Data were expressed as box and whisker plots and tested for statistical significance by the Mann-Whitney $\mathrm{U}$ test (Unistat, Unistat Ltd).

\section{Western blot analysis}

Either tissue cyst antigen (10000 tissue cysts/gel) or tachyzoites $\left(1.4 \times 10^{7}\right.$ parasites/gel $)$ were heated at $100^{\circ} \mathrm{C}$ in reducing buffer for $10 \mathrm{~min}$ before separation on a polyacrylamide $12.5 \%$ gel [14]. After SDS-PAGE separation proteins were transferred electrophoretically to nitrocellulose at $400 \mathrm{~mA}$ for $1 \mathrm{~h}$. Filters were cut into strips and blocked for $1 \mathrm{~h}$ at room temperature with non-fat milk $5 \%$ in Tween-Tris buffered saline (TTBS; $1 \mathrm{M}$ Tris $\mathrm{HCl}, \mathrm{NaCl} 0.9 \%$, Tween $200.1 \%$ ). After three washes in TTBS ( 5 min, RT) individual strips were incubated with patient sera diluted 1 in 100 in TTBS containing goat serum $10 \%$. Incubation was for 15 min at RT with continual rocking. Strips were washed as described previously and incubated with the second antibody, biotinylated goat anti-human IgG whole molecule (Amersham) diluted 1 in 1000 in goat serum $10 \%$ in TTBS for $15 \mathrm{~min}$ at RT. Again the strips were washed and then incubated with strepavidinalkaline phosphatase conjugate (Boehringer Mannheim) diluted 1 in 2000 in TTBS for $15 \mathrm{~min}$ at RT. Strips were washed four times and antigens were visualised with nitroblue tetrazolium chloride/5-bromo-4-chloro3-indolyl phosphate (NBT/BCIP) substrate following the manufacturer's instructions.

\section{Results}

A total of 187 sera was studied by both dye test and tissue cyst ELISA (Table 1).

Table 1. Patient sera submitted to quantitative evaluation

\begin{tabular}{clc}
\hline Group & Patient status & Number of sera \\
\hline 1 & Immunocompetent & 20 \\
& T. gondii seronegative & 12 \\
2 & Immunocompetent & \\
& T. gondii dye test positive & 7 \\
$\quad$ & IgM seronegative & \\
3 & Immunocompetent & 66 \\
& T. gondii dye test positive & 22 \\
4 & IgM seropositive & 36 \\
5 & Active ocular toxoplasmosis & \\
6 & Quiescent ocular toxoplasmosis & 24 \\
7 & HIV seropositive & \\
7 & Active toxoplasmosis & 187 \\
Total & Quiescent toxoplasmosis & \\
\hline
\end{tabular}

Dye test values for each of the $T$. gondii seropositive groups are shown in Fig. 1. There was no statistical difference between immunocompetent $T$. gondii-seropositive patients and either ocular or HIV-positive $T$. gondii seropositive samples. Similarly, there was no statistical difference between the results in active and quiescent toxoplasmosis in either ocular or HIVpositive samples.

The results of the $T$. gondii tissue cyst-specific ELISA are shown in Fig. 2. Immunocompetent $T$. gondii seropositive patients demonstrated a significantly increased antibody response to cyst antigens when compared to all other groups of sera $(p<0.0001)$. There was no statistical difference in $T$. gondii tissue cyst-specific ELISA results between $T$. gondii-specific IgM positive $(\mathrm{n}=7)$ and $T$. gondii-specific $\operatorname{IgM}$ negative $(\mathrm{n}=12)$ immunocompetent sera. Patients with ocular disease represented a single statistical population with no significant difference between the active and quiescent states and the antibody titres were not significantly different from immunocompetent $T$. gondii seronegative samples. HIV-positive sera show a trend to two distinct populations $(\mathrm{p}=0.036)$. Sera from HIV-positive patients with active disease were not significantly different from immunocompetent $T$. gondii seronegative samples, but those samples taken during quiescence had a raised antibody titre $(\mathrm{p}<0.001)$ as compared to immunocompetent $T$. gondii seronegative samples.

Western blot analysis was performed on 57 HIVpositive, $T$. gondii seropositive sera; 15 of these sera were from patients with quiescent $T$. gondii infection and 42 from patients with active cerebral disease. Controls included in each blot were HIV-positive $T$. gondii seronegative sera. Figs. 3 and 4 are distribution plots of the tissue cyst (Fig. 3) and tachyzoite (Fig. 4) antigens recognised by each serum and a representative blot is shown for each (Figs. 5 and 6). The profile of the tissue cyst and tachyzoite antigens recognised by sera from patients with active disease was similar to that of patients with quiescent disease. Tissue cyst antigens recognised by more than one-third of the sera were recorded at $65,57,49,47,36,28$ and $26 \mathrm{kDa}$ in both quiescent and active disease sera. An $18-\mathrm{kDa}$ antigen was recognised in 5 of 15 quiescent disease sera. Tachyzoite antigens recognised by more than one-third of sera were $75,51,44,33,30$ and $25 \mathrm{kDa}$ in quiescent sera only, 49 and $32 \mathrm{kDa}$ in active disease only and $66,38,28,22$ and $21 \mathrm{kDa}$ in both patient groups.

No single antigen was recognised by all sera in one patient group. Variation was noted between immunocompetent $T$. gondii sero-positive sera. The fact that no single antigen or antigen profile was observed to correlate with the majority of individuals within a single patient group suggests the absence of an antigen of diagnostic or prognostic value. 


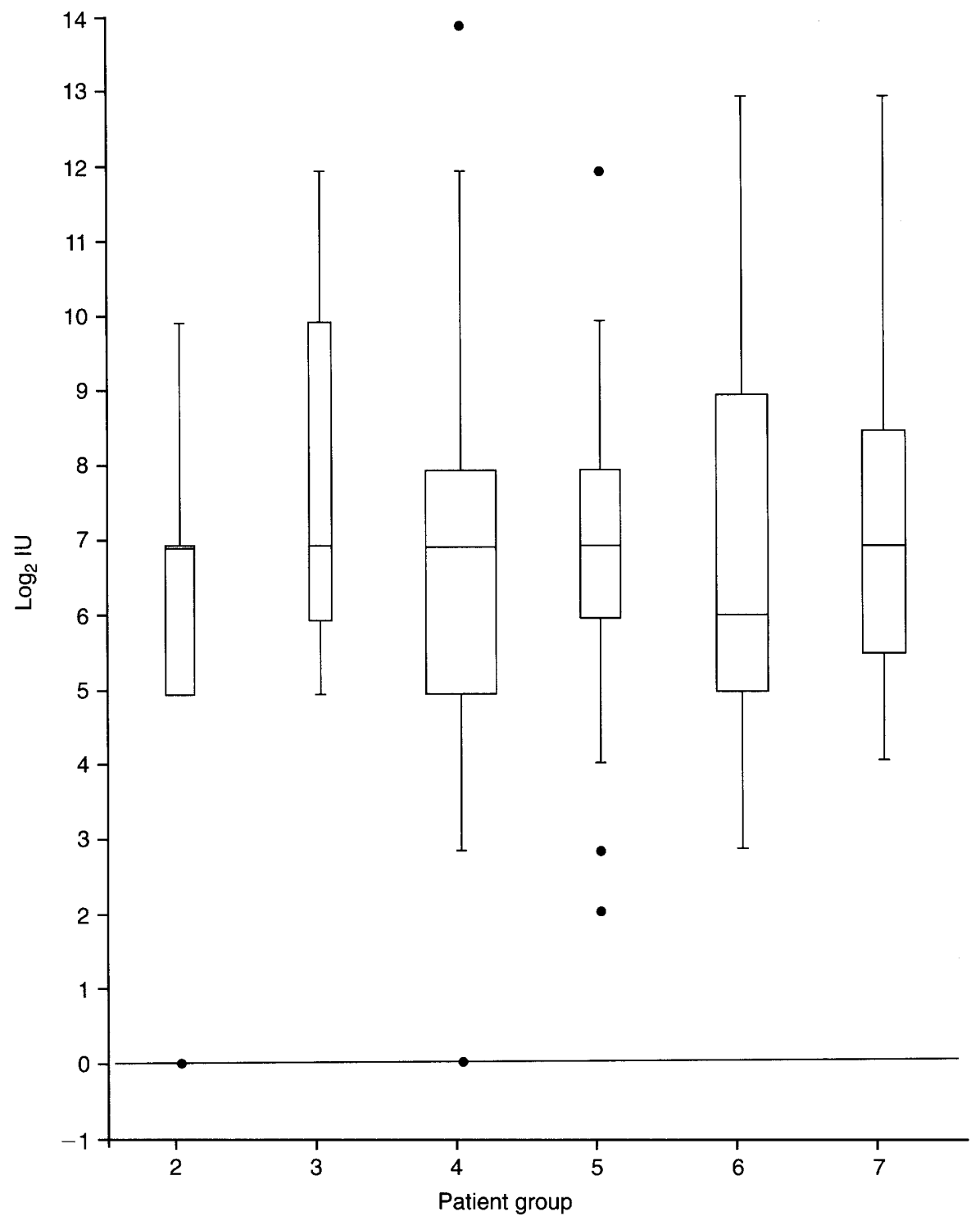

Fig. 1. Sabin-Feldman dye test data $\left(\log _{2} \mathrm{IU}\right)$ for all $T$. gondii seropositive patients. Patient groups as Table 1 . Box and whisker plot: bottom line of box = lower quartile; middle line of box = median; top line of box $=$ upper quartile; whiskers $=$ quartile minus 1.5 times inter-quartile range; $\mathbf{O}=$ outlying results.

\section{Discussion}

This study reports an investigation of sera from patients with reactivating toxoplasmosis compared to those in quiescence. The gold standard Sabin-Feldman dye test confirmed the presence of $T$. gondii-specific antibody, but did not differentiate active from quiescent disease either in HIV-positive or ocular disease patients, confirming previous work [15]. The dye test recognises complement fixing antibody, primarily $\operatorname{IgG}$ but also IgM, reactive with tachyzoites. Following primary exposure the dye test may remain raised for many years [16]. The fact that, in all patient types studied, dye test results were statistically the same may be interpreted as reflecting the continued exposure of the host immune system to tachyzoite-derived immunogens, associated with the controlled turnover of tissue cysts and sustained trickle release of tachyzoites for immune priming or promoting prolonged B-cell memory, or both. Although the dye test data from immunocompetent patients shows no statistical difference between IgM-positive and IgM-negative patients, the trends in these two populations are as may be expected for mixed populations of IgG and IgM titres. In immunocompetent $T$. gondii-specific IgM-positive patients, the distribution is skewed to lower titres, reflecting the high IgM titre and the reduced contribution of IgG. In immunocompetent $T$. gondii-specific IgM-negative paitents, the increased contribution of specific IgG is reflected in a distribution skewed to higher dye test results. The relative insensitivity of the quantitative dye test to differentiate between chronic and acute infections may reflect antigenic variation in UK strains compared to the RH strain of $T$. gondii on which the assay is based.

It is proposed that in the immunocompetent host the natural history of tissue cyst development and 


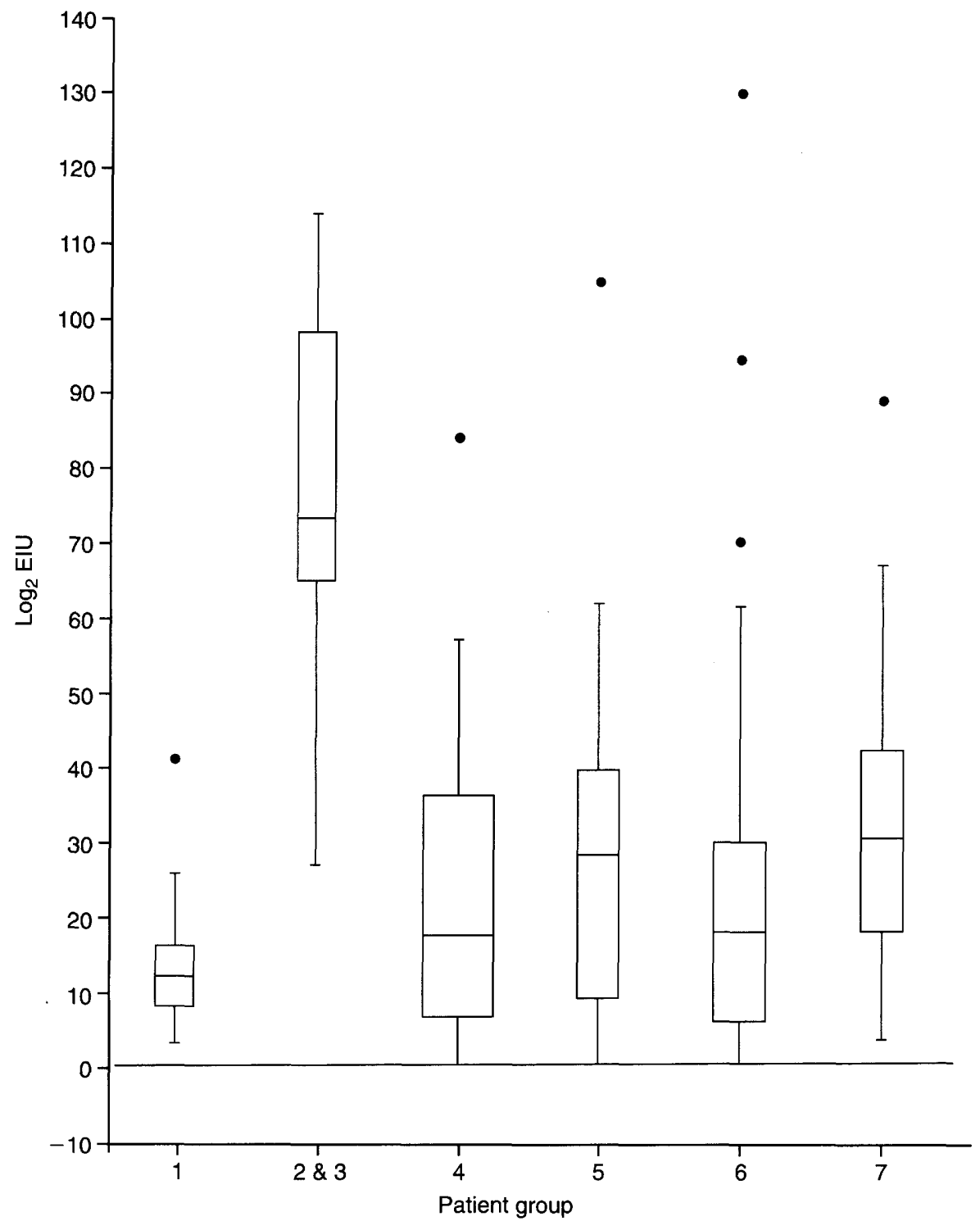

Fig. 2. Tissue cyst ELISA data ( $\log _{2}$ EIU) for all patient groups. Details as for Fig. 1 .

rupture turnover is under some degree of immune control, but that in the immunocompromised host this equilibrium is disturbed and reactivation disease results. The concept of the tissue cyst population as being in a state of dynamic equilibrium is supported by histological observation [17], studies in tissue culture [18] and the demonstration of parasitaemia by gene amplification methods in the absence of focal encephalitis $[19,20]$. This model challenges the commonly held view of reactivation as a single dramatic event and proposes that the clinical presentation of reactivating disease indicates previous episodes of subclinical reactivation before the development of demonstrable pathology. The serological data presented here support this view, as they show no marked difference in anti-tissue cyst antibody in active or quiescent toxoplasmosis from either patients with ocular disease or HIV.

The quiescent sera tested included samples from patients treated for a previous episode of active toxoplasmosis. Our definition of quiescence may be more correctly defined as 'post-treatment quiescence' and antibody responses in this group may be distinct from patients with no previous reactivation and may be more like active disease. Discrimination between these two categories would require anti- $T$. gondii antibody levels to fall in quiescence. However, dye test data and the dynamic tissue cyst model suggest that levels are maintained; furthermore, the Western blot data also demonstrate no qualitative change. To establish the precise relationship between 'post-treatment quiescence' and 'no reactivation quiescence' a prospective study following the serological outcome of congenital toxoplasmosis would be necessary. This approach would enable the identification of early reactivation events; however, ethical considerations may exclude the possibility of such a study.

In designing an ELISA specific for $T$. gondii tissue cyst antigens, the aim was to establish whether the deregulated reactivation associated with either HIV or 


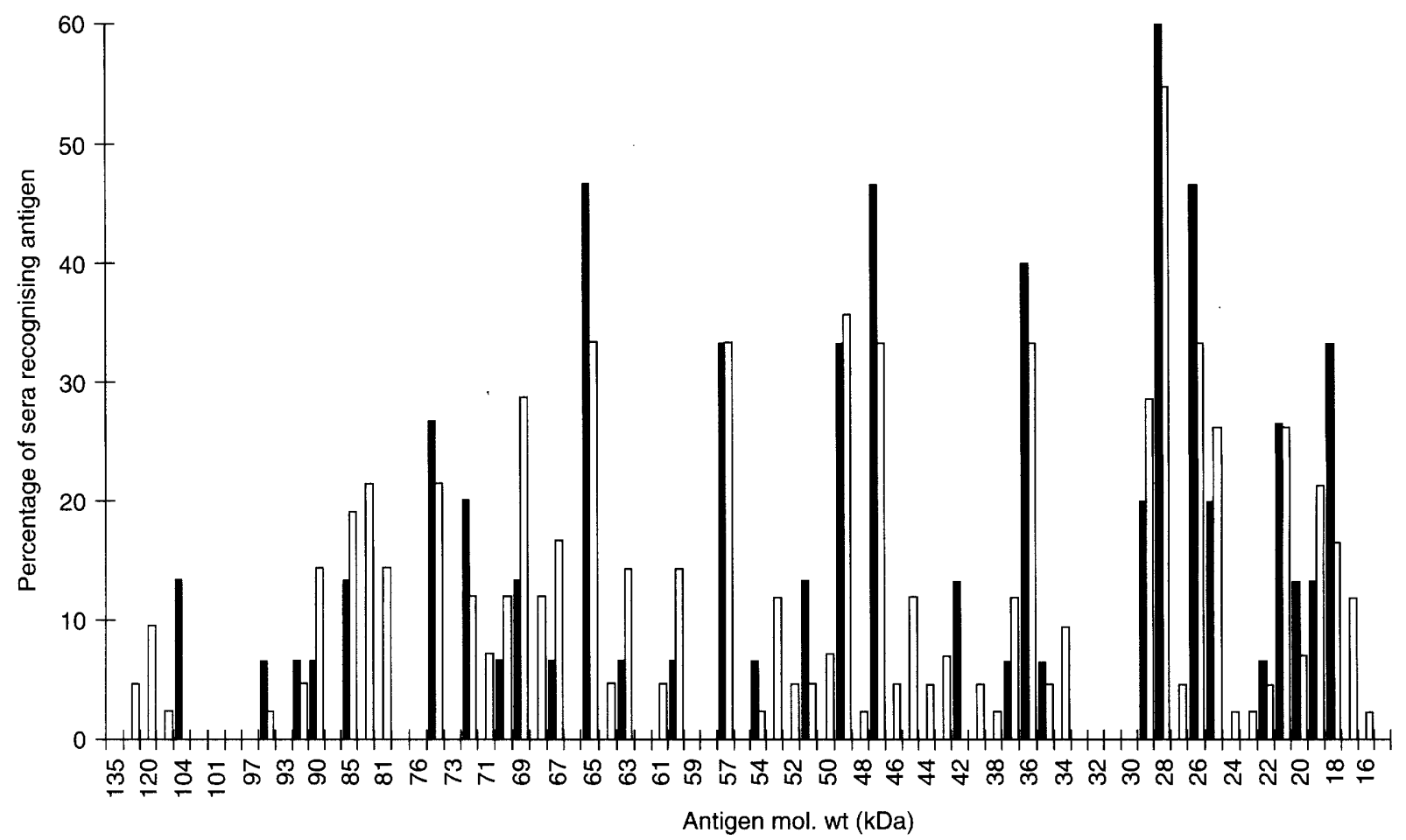

Fig. 3. Percentage recognition of tissue cyst antigens recognised by sera from HIV patients with quiescent $(n=15$; and active toxoplasmosis $(n=42 ; \square)$.

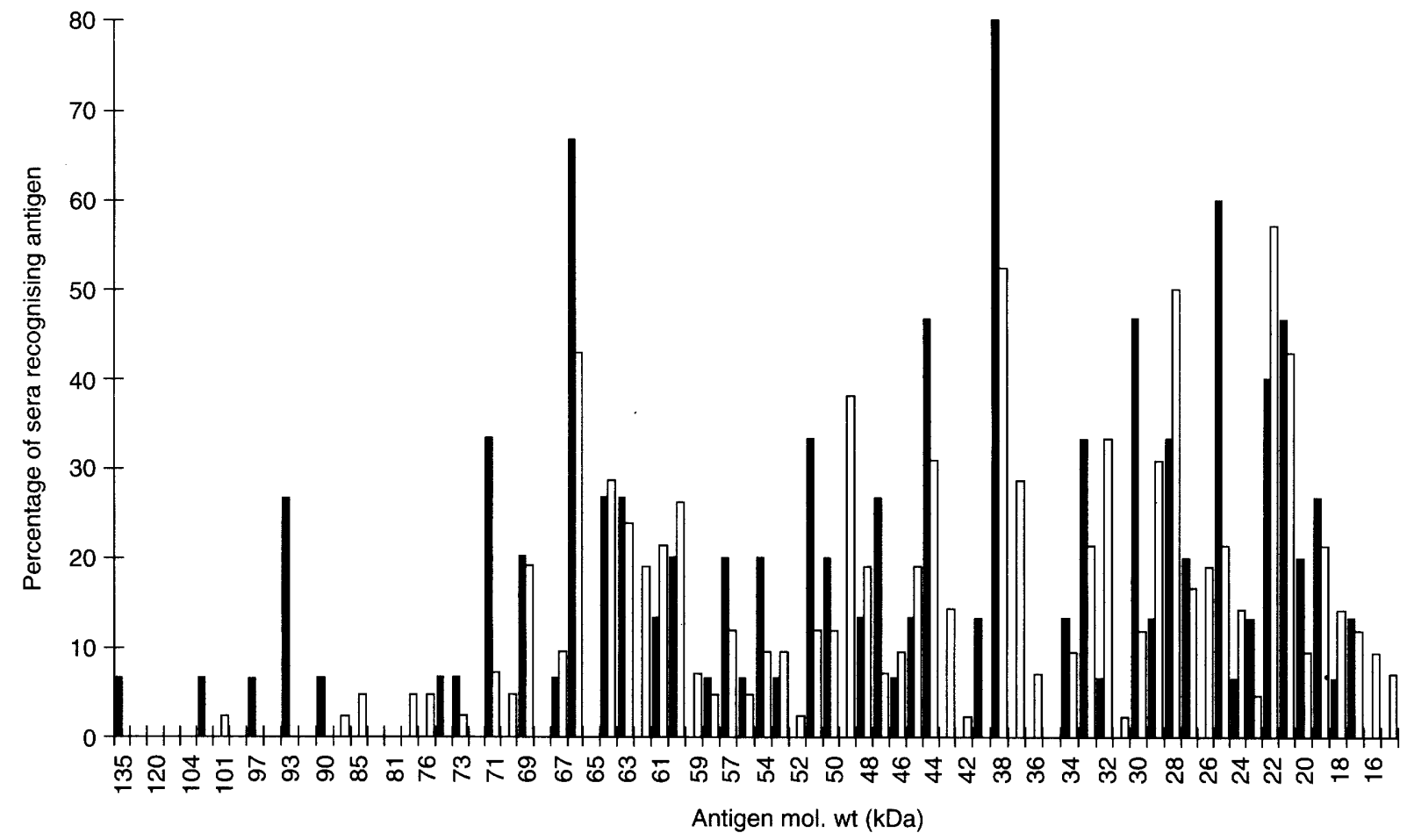

Fig. 4. Percentage recognition of tachyzoite antigens recognised by sera from HIV patients with quiescent $(n=15$; and active toxoplasmosis $(n=42 ; \square)$.

ocular disease was associated with a quantitative change in tissue cyst-specific antibody. A whole tissue cyst preparation was adopted which included tissue cyst wall, tissue cyst matrix and bradyzoite antigens. The evidence from both the present study and previous authors [21] indicates that this life cycle stage expresses fewer antigens than the tachyzoite and that the immune response to these antigens is lower. Of the sera studied, the only population to be markedly different from the others was the sera from immunocompetent $T$. gondii seropositive patients $(\mathrm{p}<0.0001)$. This group represents patients with prior 


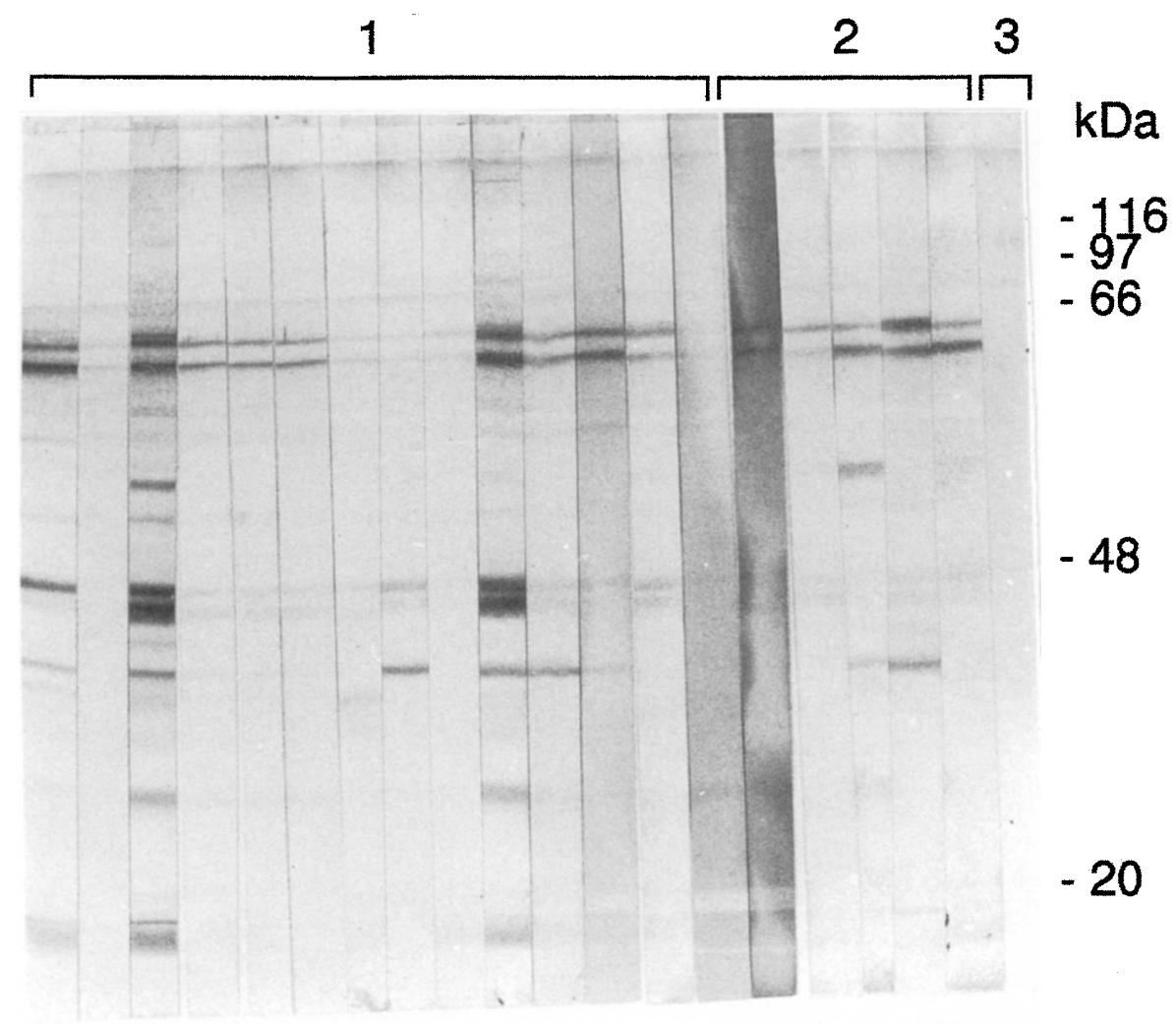

Fig. 5. Representative Western blot of $T$. gondii tissue cyst antigens screened with: 1, sera from HIV-positive patients with active toxoplasmosis; 2 , sera from HIV-positive patients with quiescent toxoplasmosis; $\mathbf{3}$, negative control serum, HIV-positive and $T$. gondii seronegative. This figure does not include sera recognising all antigens indicated in Fig. 3 , as many antigens were recognised by one-third or less of patient sera.

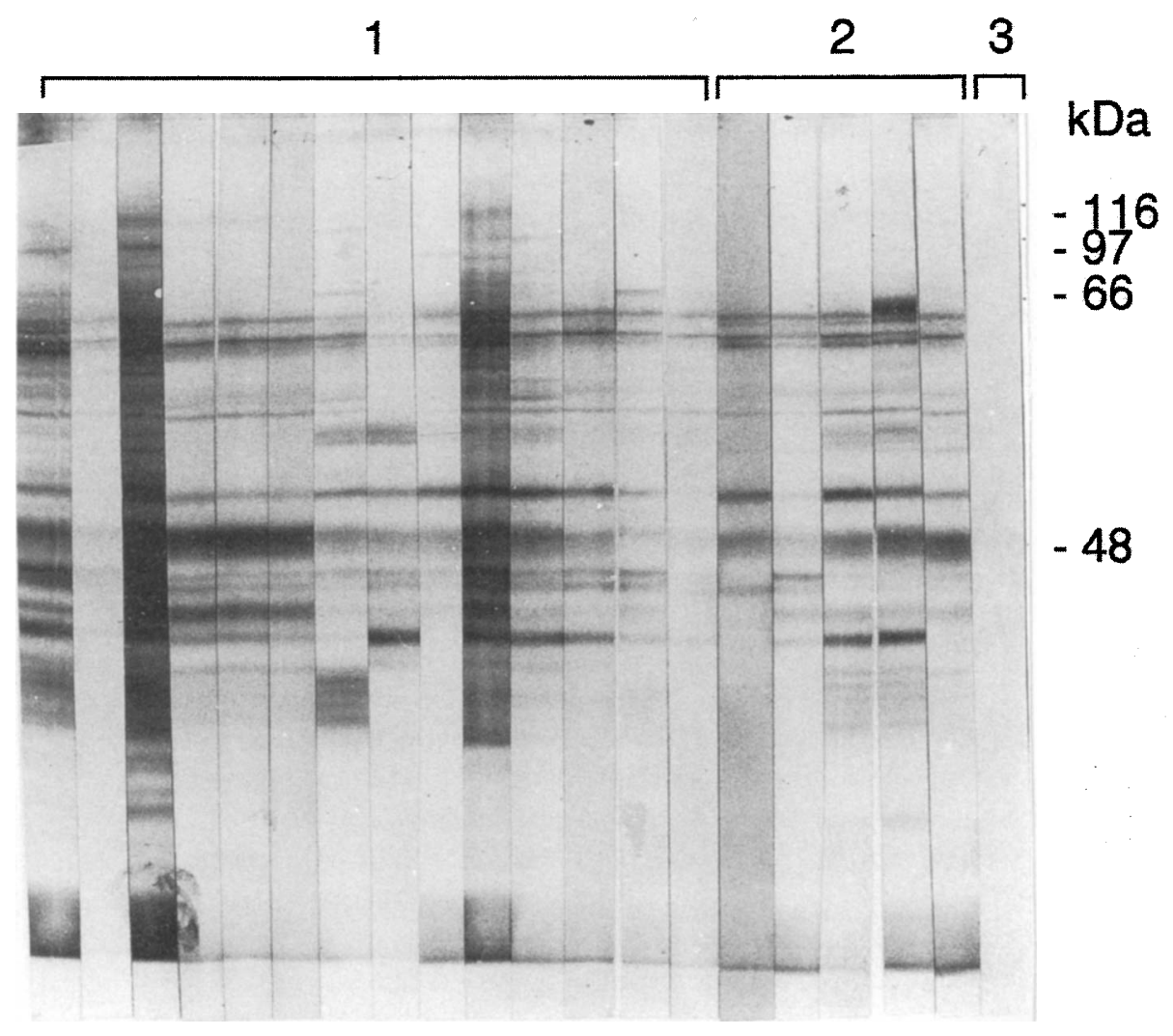

Fig. 6. Western blot of $T$. gondii tachyzoite antigens screened with the same sera as shown in Fig. 5. 
exposure to tachyzoites and the increase in anti-tissue cyst antibody level may reflect recognition of epitopes that are common to both tachyzoites and bradyzoites [5] or exposure to the intermediate steps of tachyzoitebradyzoite interconversion [6], or both, reflecting the dynamic equilibrium between tachyzoites and tissue cysts. The contribution of shared epitopes to the antitissue cyst antibody level may be addressed in future studies by the pre-absorption of sera with tachyzoites. Furthermore, tissue cysts may form early in an infection such that immunocompetent patients, even in the acute phase of infection, will have been exposed to tissue cyst antigens. Thus, patients with both recently acquired and long-standing infections will respond to tissue cyst antigens, although, as Zhang et al. [21] demonstrated, anti-tissue cyst antibody titres rise slowly in comparison to antitachyzoite antibody titres.

HIV-positive quiescent samples had raised $T$. gondii tissue cyst-specific antibody levels. However, in both populations of patients with active toxoplasmosis (HIV positive or ocular disease), levels of tissue cystspecific antibody titres were similar to those of $T$. gondii seronegative sera. Failure of patients with chronic exposure to $T$. gondii to recognise tissue cyst antigens may be a result of antigens not being exposed to the host immune system; tissue cysts are located primarily in sites regarded as being immune privileged. Furthermore, Western blot analysis of tissue cyst antigens with sera from cyst immunised mice [unpublished observations, 22] reveals six-toeight antigens, suggesting that tissue cysts display only a limited set of antigens and do not elicit a complex immune response. This observation corresponds to the histological evidence that immune infiltration occurs only after tissue cyst rupture [17] and the view that $T$. gondii has a preference for sites which are immunologically privileged [23]. Thus, the tissue cyst is concealed from host immune surveillance until the actual event of reactivation. The cyclic reactivation proposed above presents a trickle of predominantly tachyzoite antigens to the host. The principal immunogens associated with reactivation are thus tachyzoite-derived, as demonstrated by the dye test data and not tissue cyst or bradyzoite-derived as demonstrated by the ELISA. Therefore, anti-tissue cyst antigen antibody levels are not a good correlate of reactivation in AIDS.

The tissue cyst ELISA did demonstrate a significant difference in tissue cyst-specific antibody level in the two HIV-positive categories. The patients with active disease demonstrated lower titres than those in quiescence. It may be that this observation is a reflection of the immunocompromised status of the patients in the active disease group; total antibody levels in this cohort will be reduced and thus tissue cyst-specific antibody may also be proportionately depleted. However, the corresponding dye test values for this group did not reflect such a depletion (Fig. 1). The apparent disparity between tissue cyst ELISA and dye test levels may be associated with an impaired ability of HIV-positive patients to process novel antigens resulting from tisssue cyst turnover. Reactivation occurs in the period when immunosuppression of the HIV positive patient is most apparent [15]. The results may indicate that tissue cyst antigens generate only short-term immunological memory rather than the long-term memory for tachyzoite antigens demonstrated by the dye test, resulting in no apparent decrease in response to the dye test at this stage of HIV infection. Alternatively, disparity in the response of these two groups may correspond to the variation in degree of immunosuppresion between the groups. However, in this retrospective study HIV-positive sera were not $\mathrm{CD}^{+}$scored, as ethical approval had not been sought. Thus, anti-cyst antigen titre cannot be correlated with $\mathrm{CD}^{+}$count to clarify the role of the extent of immunosuppression in the difference between HIV-positive active and quiescent sera, although previous studies indicate that TE is usually associated with $\mathrm{CD}^{+}$counts $<200$ cells $/ \mathrm{mm}^{3}$ [15].

Data from patients with reactivating ocular toxoplasmosis demonstrate that tissue cyst-specific antibody levels do not differ in active and quiescent disease and indeed they are not significantly different from immunocompetent $T$. gondii seronegative sera. This may reflect the concealed nature of the tissue cyst antigens and release of antigenically distinct life cycle stages on reactivation. This study did not distinguish between patients with documented CT and those with acquired infection leading to ocular disease. However, this sample is likely to contain predominantly patients with CT, as in a UK population the majority of patients with ocular toxoplasmosis are infected as a result of congenital infection. Thus, the inability of an apparently immunocompetent population to recognise tissue cyst antigens may be a result of infection in utero followed by invasion of immune privileged sites and concealment of the parasite before the full development of the neonatal immune system. It is also possible that patients who develop ocular toxoplasmosis represent a population distinct from acquired toxoplasmosis patient groups because of either partial immunosuppression or tolerance.

The data in Fig. 2 do identify immunocompetent patients as having raised anti-tissue cyst titres. This observation may be of use in characterising genuinely latent forms of toxoplasmosis in which 'healthy carriership' is identified. This approach may enable the distinction between chronic quiescent infection and active, non-symptomatic infection where a differential diagnosis is being sort for such conditions as chronic fatigue syndrome.

Interpretation of the quantitative data indicating that chronic toxoplasmosis reflects a dynamic situation in 
which the host is exposed to a limited range of immunogens which are largely shared with tachyzoites is confirmed by the Western blot data presented here and by previous authors $[4,23]$. In this analysis of 57 patient sera, few antigens were recognised by more than one-third of the sera and no single antigen was diagnostic of quiescence or active disease. This study adopted a single strain as the source of antigen both for ELISA and Western blot. Strain variation has been demonstrated by Ware and Kasper [24] and Woodison [22]. However, the differences noted by these authors were limited and the major immunogens were conserved, such as the $36-\mathrm{kDa}$ antigen described in this study, by Woodison [22] and by Tomavo [25]. The present study demonstrated eight antigens $(65,57,49$, $47,36,28,26$ and $18 \mathrm{kDa}$ ) which are consistently recognised by one-third or more of the sera tested. This limited number of antigens is in agreement with other authors and the molecular mass of these antigens is also comparable with those described previously $[4,23,25]$.

In conclusion therefore, tissue cyst-derived antigens are not a reliable serological marker to distinguish active from post-active reactivating toxoplasmosis. The current study was performed with groups of patient sera. It is feasible that the analysis of serial samples from individuals before their first reactivation event may identify specific antigens associated with reactivation. However, the dynamic model of tissue cyst maintenance proposed here would not predict this outcome. This study failed to identify a single immunogen that was diagnostic of reactivation, but this should not preclude study of candidate molecules as they are identified through alternative strategies. However, this study provides support for the model of T. gondii tissue cysts as a dynamic population undergoing a cyclical and continuous turnover under immune control and for the proposal that reactivation reflects the deregulation of this process.

This work was funded by the MRC.

\section{References}

1. Luft BJ, Remington JS. Toxoplasmic encephalitis in AIDS. Clin Infect Dis 1992; 15: 211-222.

2. Holliman RE, Stevens PJ, Duffy KT, Johnson JD. Serological investigation of ocular toxoplasmosis. Br J Ophthalmol 1991; 75: $353-355$.

3. Suzuki Y, Remington JS. Importance of membrane-bound antigens of Toxoplasma gondii and their fixation for serodiagnosis of toxoplasmic encephalitis in patients with Acquired Immunodeficiency Syndrome. J Clin Microbiol 1990; 28: $2354-2356$.
4. Kasper LH. Identification of stage-specific antigens of Toxoplasma gondii. Infect Immun 1989; 57: 668-672.

5. Darcy $\mathrm{F}$, Charif $\mathrm{H}$, Caron $\mathrm{H}$ et al. Identification and biochemical characterization of antigens of tachyzoites and bradyzoites of Toxoplasma gondii with cross-reactive epitopes. Parasitol Res 1990; 76: 473-478.

6. Soete M, Fortier B, Camus D, Dubremetz JF. Toxoplasma gondii: kinetics of bradyzoite-tachyzoite interconversion in vitro. Exp Parasitol 1993; 76: 259-264.

7. McHugh TD, Gbewonyo A, Johnson JD, Holliman RE, Butcher PD. Development of an in vitro model of Toxoplasma gondii cyst formation. FEMS Microbiol Lett 114: 1993; 325-332.

8. Roberts CW, Alexander J. Studies on a murine model of congenital toxoplasmosis: vertical disease transmission only occurs in $\mathrm{BALB} / \mathrm{c}$ mice infected for the first time during pregnancy. Parasitology 1992; 104: 19-23.

9. Cornelissen AWCA, Overdulve JP, Hoenderboom JM. Separation of Isospora (Toxoplasma) gondii cysts and cystozoites from mouse brain tissue by continuous density-gradient centrifugation. Parasitology 1981; 83: 103-108.

10. Savva D, Morris JC, Johnson JD, Holliman RE. Polymerase chain reaction for detection of Toxoplasma gondii. $J$ Med Microbiol 1990; 32: 25-31.

11. Sabin AB, Feldman HA. Dyes as microchemical indicators of a new immunity phenomenon affecting a protozoon parasite (Toxoplasma). Science 1948; 108: 660-663.

12. Duffy KT, Wharton PJ, Johnson JD, New L, Holliman RE Assessment of immunoglobulin-M immunosorbent agglutination assay (ISAGA) for the detection of Toxoplasma specific IgM. J Clin Pathol 1989; 42: 1291-1295.

13. Balfour AH, Harford JP. Quality control and standardisation. In: Wreghitt TG, Morgan-Capner P (eds) ELISA in the clinical microbiology laboratory. London, Public Health Laboratory Service. 1990; 36-47.

14. Laemmli UK. Cleavage of structural proteins during the assembly of the head of bacteriophage $T_{4}$. Nature 1970; 277 : $680-685$.

15. Holliman RE. Toxoplasmosis and the acquired immunedeficiency syndrome. J Infection 1988; 16: 121-128.

16. Suzuki Y, Thulliez P, Remington JS. Use of acute-stagespecific antigens of Toxoplasma gondii for serodiagnosis of acute toxoplasmosis. $J$ Clin Microbiol 1990; 28: 1734-1738.

17. Frenkel JK, Escajadillo A. Cyst rupture as a pathogenic mechanism of toxoplasmic encephalitis. Am J Trop Med Hyg 1987; 36: 517-522.

18. McHugh TD, Holliman RE, Butcher PD. The in vitro model of tissue cyst formation in Toxoplasma gondii. Parasitol Today 1994; 10: 281-285.

19. Filice GA, Hitt JA, Mitchell CD, Blackstad M, Sorensen SW. Diagnosis of Toxoplasma parasitemia in patients with AIDS by gene detection after amplification with polymerase chain reaction. J Clin Microbiol 1993; 31: 2327-2331.

20. Liesenfeld O, Roth A, Weinke T, Foss H-D, Hahn H. A case of disseminated toxoplasmosis - value of PCR for the diagnosis. $J$ Infection 1994; 29: 133-138.

21. Zhang YW, Fraser A, Balfour AH, Wreghitt TG, Gray JJ, Smith JE. Serological reactivity against cyst and tachyzoite antigens of Toxoplasma gondii determined by FAST-ELISA. $J$ Clin Pathol 1995; 48: 908-911.

22. Woodison G, Smith JE. Identification of the dominant cyst antigens of Toxoplasma gondii. Parasitology 1990; 100: 389-392.

23. Woodison G, Balfour AH, Smith JE. Sequential reactivity of serum against cyst antigens in Toxoplamsa infection. $J$ Clin Pathol 1993; 46: $548-550$.

24. Ware PL, Kasper LH. Strain-specific antigens of Toxoplasma gondii. Infect Immun 1987; 55: 778-783.

25. Tomavo S, Fortier B, Soete M, Ansel C, Camus D, Dubremetz JF. Characterization of bradyzoite-specific antigens of Toxoplasma gondii. Infect Immun 1991; 59: 3750-3753. 